\title{
Magnetic Hyperfine Fields of Nanoperm Alloys
}

\author{
M. Miglierini ${ }^{a, b}$, J. KOHOUT ${ }^{c}$, A. LANČOK ${ }^{d}$ AND K. ŠAfÁřOvÁ ${ }^{b}$ \\ ${ }^{a}$ Slovak University of Technology, Ilkovičova 3, 81219 Bratislava, Slovakia \\ ${ }^{b}$ Regional Centre of Advance Technologies and Materials, Palacky University \\ Šlechtitelů 11, 77146 Olomouc, Czech Republic
}

${ }^{c}$ Faculty of Mathematics and Physics, Charles University, V Holešovičkách 2, 18000 Prague, Czech Republic

${ }^{d}$ Institute of Inorganic Chemistry of the AS CR, v. v. i., 25068 Husinec-řež 1001, Czech Republic

\begin{abstract}
Magnetic hyperfine fields of $\mathrm{Fe}_{90} \mathrm{Zr}_{7} \mathrm{~B}_{3}$ Nanoperm nanocrystalline alloy are characterized by ${ }^{57} \mathrm{Fe}$ Mössbauer spectrometry and ${ }^{57} \mathrm{Fe}$ NMR as well as by magnetic force microscopy. ${ }^{57} \mathrm{Fe}$ NMR enables to distinguish a broad signal of iron atoms located in a residual amorphous matrix from a narrow one which belongs to Fe in nanograins. The former coincides with the distribution of hyperfine fields obtained from ${ }^{57} \mathrm{Fe}$ Mössbauer spectroscopy. In addition, it is possible to make a distinction between NMR signals of the Fe nanograins located in magnetic domains from that of the nanograins positioned in domain walls. This is confirmed by magnetic force microscopy where appearance of maze-domains is observed.
\end{abstract}

PACS: 75.50.Tt, 75.75.-c, 76.80.+y, 76.60.-k, 68.37.Rt

\section{Introduction}

Practical applications [1] of nanocrystalline alloys obtained from amorphous precursors by controlled annealing stem from their advantageous soft magnetic properties [2]. To benefit from their unique magnetic parameters, we have to know their structural arrangement in detail. In addition, these materials are available in a form of ribbons with noticeable differences between the bulk and the surfaces of the ribbons [3]. Conventional magnetic measurements provide integral information over different structural components contained in the nanocrystalline alloys (i.e., nanocrystalline grains, amorphous residual matrix, and interconnecting phase). This contribution aims in characterization of magnetic hyperfine fields by employing local probing techniques like ${ }^{57} \mathrm{Fe}$ Mössbauer and ${ }^{57} \mathrm{Fe}$ nuclear magnetic resonance (NMR) spectroscopy. Surface sensitive magnetic force microscopy (MFM) is also employed. This work extends our recent studies $[4,5]$.

\section{Experimental details}

Amorphous precursors of $\mathrm{Fe}_{90} \mathrm{Zr}_{7} \mathrm{~B}_{3}$ Nanoperm alloy were prepared by rapid quenching on a rotating wheel. As-quenched ribbons (sample $A$ ) were annealed in vacuum to produce nanocrystalline samples: $510^{\circ} \mathrm{C} / 10 \mathrm{~min}$ (sample $B$ ) and $620^{\circ} \mathrm{C} / 80 \mathrm{~min}$ (sample $C$ ). Heating conditions were chosen according to the results of differential scanning calorimetry [6].

${ }^{57} \mathrm{Fe}$ Mössbauer spectra were collected with a ${ }^{57} \mathrm{Co} / \mathrm{Rh}$ source in transmission geometry and by detection of con- version electrons (CEMS). The latter scans surface regions down to the depth of $200 \mathrm{~nm} .{ }^{57} \mathrm{Fe}$ NMR experiments were performed in zero magnetic fields by the spin echo method. MFM images were recorded by NTEGRA Aura system in semicontact mode using tips with nominal radius of $30 \mathrm{~nm}$.

\section{Results and discussion}

${ }^{57} \mathrm{Fe}$ Mössbauer effect experiments show that the as-quenched $\mathrm{Fe}_{90} \mathrm{Zr}_{7} \mathrm{~B}_{3}$ alloy (sample $A$ ) is paramagnetic at room temperature while it is ferromagnetic at $4.2 \mathrm{~K}$. Corresponding spectra in Fig. 1 exhibit distribution of quadrupole splitting $P(\mathrm{QS})$ and distribution of magnetic hyperfine fields $P(B)$, respectively. At low temperature, magnetically different regions of the resonant ${ }^{57} \mathrm{Fe}$ nuclei can be identified in the amorphous structure as demonstrated by three individual spectral components and their $P(B)$ distributions. In the latter, comparison of $P(B)$ obtained from the Mössbauer spectrometry (line) and from NMR (full symbols) is presented. A satisfactory coincidence is obtained within the inspected region of magnetic hyperfine fields.

After annealing, bcc-Fe nanocrystalline grains have evolved inside the residual amorphous matrix. The Mössbauer spectra of sample $C$ taken in transmission geometry and CEMS together with distributions of magnetic hyperfine fields are shown in Fig. 2. Three main spectral components which represent different structural arrangements can be distinguished: amorphous residual phase (AM), crystalline phase (CR), and interfacial regions (IF). The latter represents bcc-Fe atoms that are 

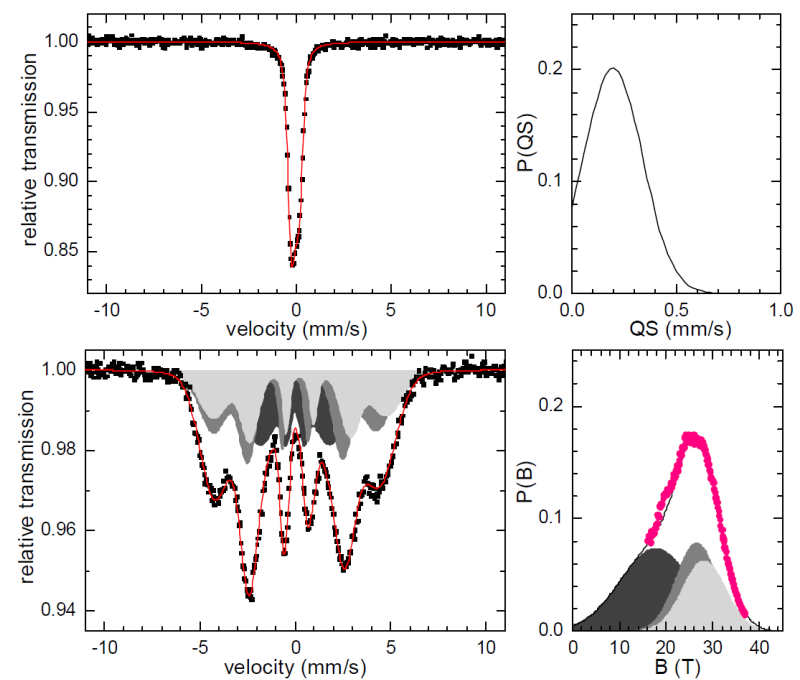

Fig. 1. Mössbauer spectra (left) and corresponding distributions of hyperfine parameters (right) of sample $A$ taken at $300 \mathrm{~K}$ (top) and $4.2 \mathrm{~K}$ (bottom). See also text.
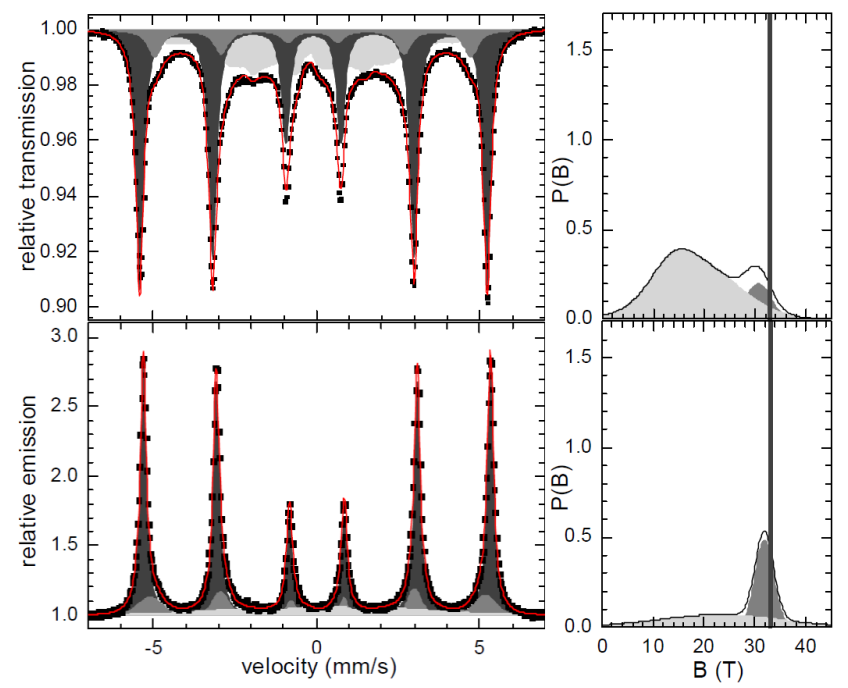

Fig. 2. Mössbauer spectra (left) and distributions of magnetic hyperfine field (right) of sample $C$ taken in transmission (top) and CEMS (bottom). Spectral components correspond to AM (light grey), CR (dark grey), and IF (grey).

located on the surface of nanocrystalline grains and are in direct contact with AM.

${ }^{57}$ Fe NMR spectra of sample $A$ are shown in Fig. 3. Obvious differences between NMR spectrum from AM and CR are demonstrated in Fig. 3a by broad and narrow lines, respectively. The latter was taken at $4.2 \mathrm{~K}$ and also at $300 \mathrm{~K}$ (Fig. 3b). Samples for NMR experiments were enriched in ${ }^{57} \mathrm{Fe}$ nuclei to provide stronger signal. These samples, however, contained $c a .2$ vol.\% of $\mathrm{CR}$ already after the preparation. Magnetic hyperfine
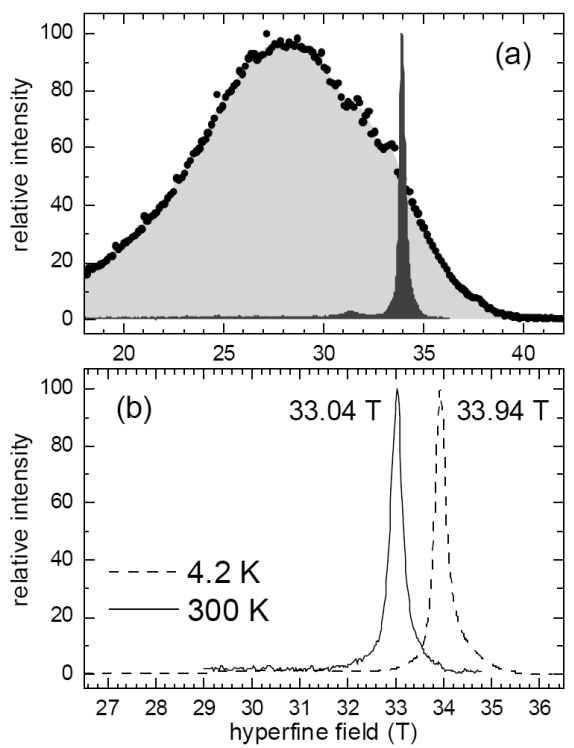

Fig. 3. ${ }^{57}$ Fe NMR spectra of sample $A$ : broad signal (light grey) - AM, narrow signal (dark grey) - CR (a), signal from nanograins taken at $300 \mathrm{~K}$ and $4.2 \mathrm{~K}$ (b).

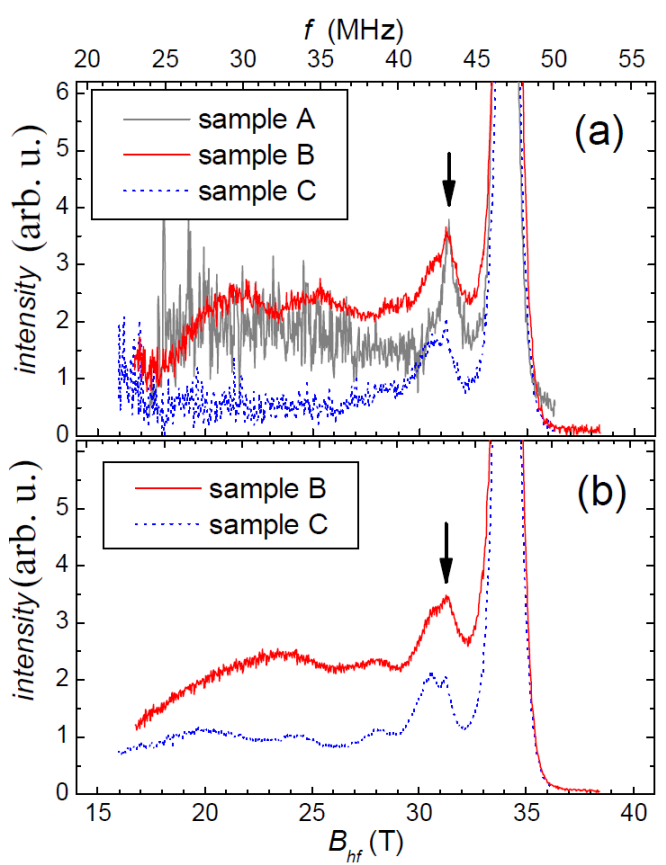

Fig. 4. ${ }^{57} \mathrm{Fe} \mathrm{NMR}$ spectra of ${ }^{57} \mathrm{Fe}_{90} \mathrm{Zr}_{7} \mathrm{~B}_{3}$ alloys corresponding to magnetic domains (a) and to domain walls (b).

fields from narrow NMR signal ascribed to nanograins fully correspond to values obtained from the Mössbauer spectrometry.

NMR spectra of CR can be inspected to more details as shown in Fig. 4. Using different RF-power, it is possible to differentiate a signal from magnetic domains (Fig. 4a) 
from that of domain walls (Fig. 4b). The spectra are normalized to 100 a.u. according to the intensity of the principal peak. It is noteworthy that no NMR signal from domain walls is observed in sample $A$. As mentioned above, in this as-quenched alloy only traces of bcc-Fe nanocrystals have been found. Because the amorphous matrix is predominantly paramagnetic, almost no magnetic domains have evolved and the corresponding spectrum in Fig. 4a is quite noisy and was recorded with difficulties.

The $y$-scale in Fig. 4 is enlarged to underline the deviations in spectral shapes of the neighbouring satellite peaks. The arrows mark positions of NMR signal which correspond to ${ }^{57} \mathrm{Fe}$ nuclei that have about $0.2 \% \mathrm{Zr}$ in their first coordination shell [4]. This is, however, by no means enough to explain the differences in magnetic hyperfine fields of CR and IF components derived from the Mössbauer spectra.

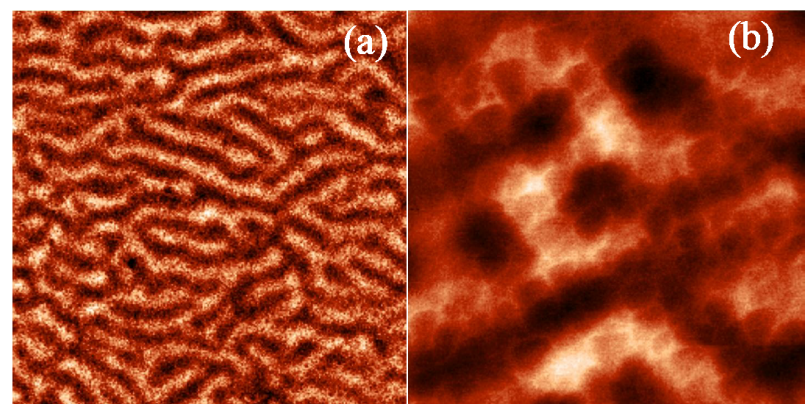

Fig. 5. MFM images of sample $C$ taken from the air side. Size of the image is $20 \times 20 \mu \mathrm{m}^{2}$ (a), $5 \times 5 \mu \mathrm{m}^{2}$ (b).

Positions of bcc-Fe nanograins in magnetic domains and domain walls are documented in Fig. 5 where MFM images acquired from the air side of sample $C$ are illustrated. One can observe nicely developed maze (finger-print) magnetic domains. Nanocrystals of bcc-Fe cover the entire surface.

\section{Conclusions}

Formation of bcc-Fe nanoparticles in originally amorphous $\mathrm{Fe}_{90} \mathrm{Zr}_{7} \mathrm{~B}_{3}$ alloy gives rise to magnetic transformation from paramagnetic to ferromagnetic arrangement even at room temperature. This can be uniquely identified by the help of both ${ }^{57} \mathrm{Fe}$ Mössbauer and ${ }^{57} \mathrm{Fe}$ NMR spectroscopy providing distributions of magnetic hyperfine fields that correspond to amorphous regions inside the alloy. On the other hand, NMR enables to inspect spectral lines of nanocrystalline grains to more details. It is even possible to distinguish NMR signal coming from ${ }^{57} \mathrm{Fe}$ nuclei located in magnetic domains from those positioned in domain walls. This is confirmed by MFM. Here, the appearance of maze-domains is related to the overcoming of demagnetizing effects in these regions by the magnetoelastic energy. The latter arises from internal stresses introduced during crystallization [7].

\section{Acknowledgments}

This work was supported by the Operational Program Research and Development for Innovations - European Regional Development Fund (CZ.1.05/2.1.00/03.0058), the Operational Program Education for Competitiveness - European Social Fund (project CZ.1.07/2.3.00/20.0017) and the grants 1M6198959201, MSM0021620834, VEGA 1/0286/12, P204/10/0035, AV0Z40320502, and SK-PL-0013-09.

\section{References}

[1] G. Herzer, Phys. Scr. T 49, 307 (1993).

[2] K. Suzuki, A. Makino, A. Inoue, T. Masumoto, J. Appl. Phys. 70, 6232 (1991).

[3] M. Pavúk, M. Miglierini, M. Vůjtek, M. Mašlán̆, R. Zbořil, Y. Jirásková, J. Phys., Condens. Matter 19, 216219 (2007).

[4] M. Miglierini, A. Lančok, J. Kohout, Appl. Phys. Lett. 96, 211902 (2010).

[5] M. Miglierini, K. Šafářová, Acta Phys. Pol. A 118, 840 (2010).

[6] S. Stankov, M. Miglierini, A.I. Chumakov, I. Sergueev, Y.Z. Yue, B. Sepiol, P. Svec, L. Hu, R. Rüffer, Phys. Rev. B 82, 144301 (2010).

[7] K. Suzuki, D. Wexler, J.M. Cadogan, V. Sahajwalla, A. Inoue, T. Masumoto, Mater. Sci. Eng. A 226-228, 586 (1997). 